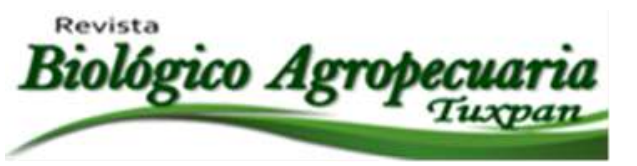

\title{
Uso de fungicidas en el manejo postcosecha de limón persa (Citrus Latifolia)
}

Use of fungicides in the postcose management of persian lemon (Citrus Latifolia)

Dávila Lezama María del Rosario ${ }^{1}$, Lorenzo Flores, Néstor Manuel ${ }^{1}$, Ramírez Hernández, Teresita ${ }^{1}$, Ángel Lara, María Alva ${ }^{1}$, Real Garrido, Carlos Jesús ${ }^{1}$

${ }^{1}$ Facultad de Agronomía de la Universidad Autónoma de Sinaloa

${ }^{\bowtie}$ Autor para correspondencia: cobicruz@hotmail.com

Recibido: $15 / 09 / 2018$

Aceptado: 15/11/2018

\section{RESUMEN}

Estudios realizados, han identificado que los hongos responsables que limitan la vida de anaquel de los cítricos son principalmente: Penicillium digitatum (55-80\%); Penicillium italicum (2-30\%); Alternaria citri y A. alternata (8-15\%); Botrytis cinerea (8-20\%): Colletotrichum gloesporioides (2.5-6\%); Geotrichum candidum (2-3\%); Rhizopus stolonifer y R. oryzae (1-3\%); Phytophtora citrophtora (2\%) (Salvador et al., 2007). El objetivo del experimento Evaluar la efectividad de dos fingicidas para el control de enfermedades provocadas por hongos en limón persa (Citrus latifolia) en postcosecha. El Proyecto se realizó en Cuajilote, Cuitláhuac, Ver. Trasladando las muestras al laboratorio general número 4 de la Facultad de Ciencias Biológicas y Agropecuarias, región Orizaba-Córdoba, de la Universidad Veracruzana. Los tratamientos donde se aplicaron los fungicidas Bankit Gold® (Azoxystrobin + Fludioxonil) y Magnate Sulphate® (Imazalil) en limón persa (Citrus latifolia) en el proceso de postcosecha, no tuvieron presencia de patógenos que provocan daños en el fruto por lo cual los fungicidas cumplieron con su objetivo, sin embargo, el tratamiento 1 (testigo absoluto) tuvo presencia del patógeno Penicillium spp. en su evaluación a los 30 DDA, esto, basándonos en los resultados de los análisis microbiológicos de limón persa (Citrus latifolia), la contaminación por Penicillium spp. probablemente fue en el almacenamiento del limón persa (Citrus latifolia). Respecto a los resultados de las propiedades fisicoquímicas están dentro los parámetros de calidad.

Palabras clave: Cítricos, hongos, Limón.

\section{ABSTRACT}

Studies have identified that the responsible fungi that limit the shelf life of citrus fruits are mainly: Penicillium digitatum (55-80\%); Penicillium italicum (2-30\%); Alternaria citri and A. alternata (8$15 \%)$; Botrytis cinerea (8-20\%): Colletotrichum gloesporioides (2.5-6\%); Geotrichum candidum (2$3 \%)$; Rhizopus stolonifer and R. oryzae (1-3\%); Phytophtora citrophtora (2\%) (Salvador et al., 2007). The objective of the experiment to evaluate the effectiveness of two fingicides for the control of diseases caused by fungi in Persian lemon (Citrus latifolia) in postharvest. The Project was carried out in Cuajilote, Cuitláhuac, Ver. Translating the samples to the general laboratory number 4 of the Faculty 
of Biological and Agricultural Sciences, Orizaba-Córdoba region, of the Universidad Veracruzana. The treatments where Bankit Gold $®$ fungicides (Azoxystrobin + Fludioxonil) and Magnate Sulphate ${ }^{\circledR}$ (Imazalil) were applied in Persian lemon (Citrus latifolia) in the post-harvest process, did not have presence of pathogens that cause damage to the fruit. fungicides fulfilled their objective, however, treatment 1 (absolute control) had presence of the pathogen Penicillium spp. in its evaluation at 30 DDA, this, based on the results of the microbiological analysis of Persian lemon (Citrus latifolia), the contamination by Penicillium spp. It was probably in the storage of the Persian lemon (Citrus latifolia). Regarding the results of the physicochemical properties are within the quality parameters.

Keywords: Citrus, fungi, Lemon

\section{INTRODUCCIÓN}

Estudios realizados, han identificado que los hongos responsables que limitan la vida de anaquel de los cítricos son principalmente: Penicillium digitatum (55-80\%); Penicillium italicum (2-30\%); Alternaria citri y A. alternata (8-15\%); Botrytis cinerea (8-20\%): Colletotrichum gloesporioides (2.5-6\%); Geotrichum candidum (2- 3\%); Rhizopus stolonifer y $R$. oryzae (1-3\%); Phytophtora citrophtora (2\%) (Salvador et al., 2007). Las enfermedades de postcosecha provocadas por estos hongos, son de gran interés, debido que son responsables de la pérdida del $40 \%$ de los alimentos producidos en el mundo (FAO, 2012), diversas investigaciones han desarrollado una gama de tratamientos para disminuir estas pérdidas, como son: Irradiaciones, tratamientos con calor (Palou, 2007), sin embargo, se ha comprobado que la utilización de fungicidas comerciales para el control de estos patógenos que provocan las podredumbres, frecuentemente son usados en distintas zonas citrícolas, cabe destacar que en postcosecha solo algunos de ellos como el benomilo o el difenilo han sido prohibidos. Por el contrario, nuevos productos están siendo evaluados y registrados. Entre los fungicidas más utilizados en postcosecha de cítricos se encuentran el imazalil (IMZ), el tiabendazol
(TBZ), el ortofenilfenato sódico (SOPP), todos ellos con una eficacia media o alta contra Penicillium spp (Pássaro et al., 2012).

El objetivo de este trabajo fue Evaluar la efectividad de 2 fungicidas en la inhibición de presencia de enfermedades provocadas por hongos en limón persa (Citrus latifolia) en postcosecha. La investigación se realizó iniciando con la cosecha de limón en la parcela ubicada en Cuajilote, Cuitláhuac, Ver., trasladándolo al laboratorio general número 4 de la Facultad de Ciencias Biológicas y Agropecuarias, región Orizaba-Córdoba, de la Universidad Veracruzana, en la Congregación de Peñuela, Municipio de Amatlán de los Reyes, Ver.

Las cajas con limón persa se recibieron el día 8 de marzo del 2018, al llegar la materia prima al laboratorio se procedió a realizar el empaque. El análisis microbiológico se realizó para determinar la presencia de patógenos, empleando como medio de cultivo agar de papa, específico para desarrollo de hongos. El análisis fisicoquímico de $\mathrm{pH}$, grados brix y acidez titulable se le realizo al jugo de limón persa en fresco, el jugo fue obtenido con exprimidores. El limón persa fue sanitizado con inmersión en agua con hipoclorito de sodio al 4 $\%$ durante 5 minutos en bandejas de plástico. Se tomó el $\mathrm{pH}$ del agua con hipoclorito de 
sodio al inicio de la sanitización el cual fue de 9.3 que al terminar la sanitización el $\mathrm{pH}$ fue de 8.4. Se prepararon los fungicidas Bankit Gold® y Magnate Sulphate ${ }^{\circledR}$ a las dosis de 3, 4 y 5 $\mathrm{ml} /$ litro de cera y $7.5 \mathrm{gr} /$ litro de cera (goma resina vegetal, poliricinoleato de gliceroles, oleato dimetiploxiloxano, agua descalcificada amoniacal, conservantes), respectivamente. Después de ser sanitizado inmediatamente fue secado manualmente con servitoallas y la aplicación de los tratamientos con cera fue con un aspersor manual. Posteriormente fueron envasados en cajas de cartón con capacidad de 18 libras, marcados con plumón para su identificación y pesados para su posterior evaluación.

Se tomó el peso de todas las cajas con limón después del encerado combinado con los tratamientos a los 0 días y posteriormente se pesó en cada evaluación que se realizó. Para la determinación del porcentaje de perdida fisiológica de peso (\%PFP), se registraron los cambios de peso de cada muestra en el día que le correspondía cada evaluación con respecto al peso inicial de su almacenamiento, utilizando una balanza granataria, Además, Se realizó el análisis fisicoquímico del jugo de limón persa en fresco por caja y por cada evaluación, el pH se evaluó con un potenciómetro de mesa marca HANNA, los grados Brix $\left({ }^{\circ} \mathrm{Bx}\right)$ con un refractómetro marca HANNA y el contenido de acidez por el método volumétrico utilizando la técnica AOAC. El contenido de acidez titulable se determinó por triplicado en \% de ácido cítrico por gramo de jugo de la pulpa de los frutos. Se realizó por titulación con $\mathrm{NaOH}$ de $0.1 \mathrm{~N}$ de acuerdo con el método establecido por la AOAC (1990). Finalmente Se realizó análisis microbiológico para determinar la presencia de hongos, el cual se llevó a cabo en cajas Petri con medio de cultivo de agar dextrosa y papa, se almacenaron en un horno de secado marca
ECOSHEL a $20{ }^{\circ} \mathrm{C}$ por 24 horas, por cada evaluación se realizó un análisis microbiológico por tratamiento.

El diseño experimental utilizado fue completamente al azar con 5 tratamientos, 3 repeticiones por tratamiento, cada repetición con 30 limones y evaluaciones a los $0,10,20$ y 30 DDA y para analizar las variables del estudio, los datos se organizaron en el programa Excel®, posteriormente con la ayuda del programa estadístico InfoStat $₫$ fueron comparados mediante una prueba de Tukey.

Los resultados muestran un efecto claro de la influencia de los tratamientos sobre este parámetro, siendo el tratamiento 3 el que menos peso perdió en las evaluaciones de los 10 y 20 días con un $1.01 \%$ y $2.60 \%$ respectivamente, el tratamiento 5 fue el que más peso perdió a los 10 y 20 días con un valor de $1.85 \%$ y $3.27 \%$ respectivamente, sin embargo a los 30 días, el tratamiento 2 mostro la menor merma en el peso, con un valor de $5.57 \%$, en contra parte el tratamiento 1 fue el que más peso perdió. Por otro lado, se pudo observar que los tratamientos 1 y 5 presentaron más del doble de pérdida de peso en todos los tratamientos estudiados al compararlos con los frutos sometidos con los tratamientos 2,3 y 4 , al final del periodo de almacenamiento, siendo evidente que el tratamiento 1, fue el peor tratamiento al perder 7.25\%. Estudios realizados por Avila et al., (2003), mencionan que la pérdida de peso está fuertemente influenciada con la temperatura de almacenamiento y al tipo de recubrimiento que se utilice, observando que las temperaturas entre 7 y $10^{\circ} \mathrm{C}$ en conjunto con los recubiertos de una formulación elaborada a base de goma de mezquite y mezcla lipídica de candelillaaceite mineral 1:1, presentaron la menor pérdida de peso del fruto, con un $3 \%$ al ser analizados a los 20 días, estos resultados son similares a los reportados por este trabajo, por 
lo que los tratamientos utilizados en este trabajo, podrían suplir a los mencionados anteriormente, siendo estos de más accesibles y de fácil manejo. Los resultados estadísticos nos muestran que no hay diferencia significativa entre tratamientos, pero si para la variable entre los días. Los resultados de grados brix en la prueba inicial (0 DDA) y la final (30 DDA), presentaron poca variación $+/-0.30$ grados, entre todos los tratamientos, sin embargo podemos mencionar que el tratamiento 5 fue el que mayor grados brix presento a los $30 \mathrm{DDA}$ con 7.6 grados y su comportamiento fue de menos a más, en contra parte el tratamiento 2 fue de más a menos grados. Alia et al., reporto en el 2011, que limones producidos en el Estado de Morelos, se caracterizan por poseer parámetros que van desde los 7.4 a 8 grados brix, valores fisicoquímicos que superan a los obtenidos en este trabajo pero Garcia et al, 2017 reporto resultados similares a los obtenidos que fueron de 7.46 con una diferencia de $+/-0.43$. Cabe resaltar que de acuerdo con la norma NMX-FF-077-1996, que hace mención de los requisitos de calidad de limón persa, para consumo humano, todos los tratamientos cumplen con estándares por arriba de la mencionada Norma. Los resultados de $\mathrm{pH}$ en jugo muestran una diferencia entre tratamientos de +/- 0.05 a los 30 días después de la aplicación de los tratamientos. Trabajos similares hechos por Puente en 2014 y Alatriste et al en 2017 presentaron una diferencia de +/0.05 , estos resultados concuerdan con los obtenidos en el este trabajo. Los análisis microbiológicos se llevaron a cabo a los 0,10 , 20 y 30 DDA, durante estas evaluaciones no se presentaron indicios de colonias de patógenos en los medios de cultivo en todos los tratamientos, sin embargo en las pruebas realizadas a los 30 DDA, el tratamiento 1 , se logró observar el crecimiento de moho azul
(Penicillium sp) en el medio de cultivo de agar de papa y dextrosa.

\section{CONCLUSIONES}

Los tratamientos donde se aplicaron los fungicidas Bankit Gold® (Azoxystrobin + Fludioxonil) y Magnate Sulphate® (Imazalil) en limón persa (Citrus latifolia) en el proceso de postcosecha, no tuvieron presencia de patógenos que provocan daños en el fruto por lo cual los fungicidas cumplieron con su objetivo, sin embargo, el tratamiento 1 (testigo absoluto) tuvo presencia del patógeno Penicillium spp. en su evaluación a los 30 DDA, esto, basándonos en los resultados de los análisis microbiológicos de limón persa (Citrus latifolia), la contaminación por Penicillium spp. probablemente fue en el almacenamiento del limón persa (Citrus latifolia). Respecto a los resultados de las propiedades fisicoquímicas están dentro los parámetros de calidad.

\section{LITERATURA CITADA}

Arias C.E.L. y Piñeros E.P.A. 2008. Trabajo de grado para obtener titulo de licenciatura. Aislamiento e identificación de hongos filamentosos de muestras de suelo de los paramos de guasca y cruz verde. Pontificia Universidad Javeriana, Bogota. 204 p.

Avila, R. M., Bosquez, E., Cortés, V., Domínguez, E., Domínguez, J., Olvera, L., \& Vernon, J. 2003. Aumento de la vida postcosecha del limón mexicano (Citrus aurantifolia Swingle) podrucido en Apatzingán, Misch., mediante uso de recubrimientos naturales a diferentes temperaturas. Red de Revistas Científicas de América Latina, El Caribe, España y Portugal, 5(2), 128-133. 
Castellano G., Ramírez R., Sindoni V.M.J., Hidalgo L.P.R., Burgos M.E., Marín R.C. y Martínez L. 2016. Efecto de la temperatura de almacenaje sobre las características organolépticas de frutos de limón persa (Citrus latifolia Tanaka). Revista Iberoamericana de Tecnología Postcosecha, 17(1), Hermosillo, México. $14 \mathrm{p}$.

De Jesús V.M. 2002. Guía Técnica Cultivo de Limón Pérsico. Programa Nacional de Frutas de El Salvador. 44 p. (Disponible en línea en http://repiica.iica.int/docs/B0217e/B0217 e.pdf)

Dussel E. 2002. Territorio y competitividad en la agroindústria en México (Vol. Primera edición). Veracruz, México. 270 p.

INIFAP. 2009. Manejo agronómico para la producción de limón persa en el estado de
Morelos. Primera Edición. Morelos, México. 27 p.

López G., Pelayo C., Castillo D., Kitinoja L. y Kader A. 2003. Técnicas de manejo poscosecha a pequeña escala: Manual para los productos hortofrutícolas. Postharvest, Technology, UCDavis, 4a Edición (8). 230 p.

Palou L. 2007. Evaluación de alternativas para el tratamiento antifúngico en poscosecha de cítricos de Producción Integrada. (200). Valencia, España. pp. 82-92.

Palou L. 2012. Situación actual del control de enfermedades de poscosecha de cítricos en España. $\quad$ Fruticultura, 23 (noviembre/diciembre), pp. 24-31.

Saucedo V.C. 2006. Perspectivas del manejo postcosecha de limas ácidas en México. Colegio de Posgraduados, Montecillos, México. 3 p.

Copyright (c) 2018 Maria del Rosario Dávila Lezama, Néstor Maruel L orenzo Flores, Teresita Ramirez Hernández, Maria Alva Ángel Lara yCarlos Jesús Real G arrido

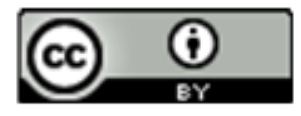

Este tex to está protegido por una licencia Creative Commons 4.0

Usted es libre para Compartir — copiar y redistribuir el material en cualquier medio o formato- y Adaptar el documento — remezclar, transformar y crear a partir del material- para cualquier propósito, incluso para fines com erciales, siempre que cumpla la condición de:

Atribución: Usted debe dar crédito a la obra original de manera adecuada, proporcionar un enlace a la licencia, e indicar si se han realizado cambios. Puede hacerlo en cualquier forma razonable, pero no de forma tal que sugiera que tiene el apoyo del licenciante o lo recibe por el uso que hace de la obra.

Resumenclelicencia - Textocompletodelalicencia 